\title{
Dynamical Probes of The Halo Mass Function
}

\author{
By C.S. Kochanek \\ Smithsonian Astrophysical Observatory \\ Harvard-Smithsonian Center for Astrophysics, MS-51 \\ 60 Garden Street, Cambridge, MA 02138 \\ ckochanek@cfa.harvard.edu
}

\begin{abstract}
We explore the relationship between the mass function of CDM halos and dynamical probes of the mass function such as the distribution of gravitational lens separations and the local velocity function. The compression of galactic halos by the cooling baryons, a standard component of

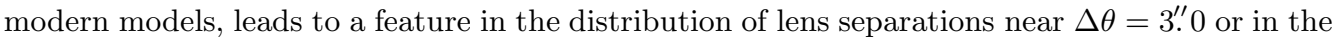
velocity function near $v_{c} \simeq 400 \mathrm{~km} \mathrm{~s}^{-1}$. The two probes of the mass function, lens separations and the local velocity function, are mutually consistent. Producing the observed velocity function of galaxies or the separation distribution using standard adiabatic compression models requires more cold baryons, an equivalent cosmological density of $\Omega_{b \text {,cool }} \simeq 0.02$ compared to a total cosmological $\Omega_{b} \simeq 0.04$, than are observed in standard accountings of the baryonic content of galaxies, $\Omega_{b, g a l} \simeq 0.006$, or our Galaxy, $\Omega_{b, \text { Gal }} \lesssim 0.015$. The requirement for a higher cold baryon density than is usually assigned to galaxies appears to be generic to models which use the standard adiabatic compression models for the transformation of the CDM halo by the cooling baryons. If real, this dynamical baryon discrepancy suggests either that we are neglecting half of the cold baryonic mass in standard galactic models (e.g. a MACHO, cold molecular or warm gas component), or that there is a problem with standard adiabatic compression models.
\end{abstract}

\section{Introduction}

In large part due to high resolution N-body simulations, the number density, spatial distribution, and properties of dark matter halos are well understood in models based on hierarchical clustering (e.g. Jenkins et al. 2000; Sheth \& Torman 1999; Navarro et al. 1996; Moore et al. 1998). The relationship of these halos to astrophysical objects is less well understood because of the modifications to the halos produced by baryonic physics and the dependence of our search and measurement techniques on their baryonic properties. While semi-analytic models of galaxy formation (e.g. Lacey \& Silk 1991, White \& Frenk 1991; Cole et al. 1994, Baugh et al. 1996, Kauffman et al. 1993, 1999, Dalcanton et al. 1997; Somerville \& Primack 1999; Benson et al. 2000; Cole et al. 2000伊) model these effects with considerable success, the results depend on detailed, parametric models for star formation and feedback processes tuned to fit the data.

We would like to have approaches for comparing the properties of halos and astrophysical objects which minimize the dependence of the comparison on star formation and luminosity. We can generally refer to these methods as dynamical probes of dark matter halos because they focus on the observational properties of the mass distribution rather than of the luminosity distribution. This approach is well-developed for massive clusters, where there are many projects designed to determine the cosmological model and the normalization of the power-spectrum by comparing the abundance of clusters with the mass function (see the reviews by Bahcall, Donahue, Rosati \& Tyson in these proceedings). Here we want to focus more on the mass scales of galaxies and on global comparisons including galaxies, groups and clusters rather than the simpler case of rich

$\dagger$ We will collectively refer to the results of semi-analytic models as SA 
clusters. The two dynamical probes we can use to relate the halo mass function to astrophysical objects are the velocity function, the distribution of halos in their circular velocity, and the separation distribution of gravitational lenses.

Although we seek tests which avoid any dependence on the luminosity of a halo, we cannot avoid the effects of the baryons. The cooling of the baryons in the lower mass halos (i.e. galaxies) and the associated adiabatic compression of the dark matter (e.g. Blumenthal et al. 1986, SA) significantly alters the density distribution of the halo and thus the properties of any dynamical probe of the halo. While these effects are included in almost all semi-analytic models, we approach the problem from a very different viewpoint. These models are also at the center of the controversy over the consistency of the cusped dark matter profiles predicted by simulations with the observed central rotation curves of galaxies (e.g. Flores \& Primack 1994, Moore 1994, de Blok et al 2001, van den Bosch et al. 2000, 2001, Salucci 2001, also Burkert, Sancisi \& Sanders in these proceedings). If there is a conflict and it is not due to an problem in the dark matter density distribution, then it must be due to a problem in either the adiabatic compression model or the assumed baryon distribution. The latter possibility is particularly interesting because we know that standard models of galaxies include only a small fraction of the available baryons.

We start in $\S 2$ with a compressed review of the models we use to determine the mass function of halos, the adiabatic compression of halos and simple cooling models for the baryons. In $\S 3$ we use these models to understand the distribution of image separations in gravitational lenses. In $\S 4$ we use the same models to study the local velocity function of galaxies and clusters. In $\S 5$ we make a final check of the consistency of the model by determining the velocity function from the gravitational lens separation distribution rather than local dynamics. Finally in $\S 6$ we outline a non-parametric approach to understanding the relation between the velocity function of galaxies and the mass function of halos. We discuss the future of dynamical probes in $\S 7$.

\section{A Very Compressed Theoretical Review}

We use a fixed $\Omega_{0}=0.3 \Lambda \mathrm{CDM}$ cosmological model with a Hubble constant of $H_{0}=$ $67 \mathrm{~km} \mathrm{~s}^{-1} \mathrm{Mpc}^{-1}$, a baryon density of $\Omega_{b}=0.04$ and a power spectrum normalized by the abundance of rich clusters $\left(\sigma_{8}=0.9\right)$. We calculated the mass function of the dark matter halos using the Press-Schechter (1974) theory combined with the Sheth \& Torman (1999) fit to the results of the Virgo simulations. Where needed, we followed Kitayama \& Suto (1996) and Newman \& Davis (2000) in modeling the distribution of halo formation times using the extended Press-Schechter theory outlined in Lacey \& Cole (1994). We neglected the problem of "halos-in-halos" (e.g. Peacock \& Smith 2001, Scoccimarro et al. 2001), as it introduces considerable complexity for very modest changes in the mass function $(\sim 10 \%$, White et al. 2001). In short, we assume the halo occupancy number is unity for galaxies and neglect accounting for the galaxies in clusters.

We used the Mo et al. (1998) model for the modifications to the mass distribution created by the cooling of the baryons and the adiabatic compression of the dark matter, although similar approaches are used in all SA studies. We assume that halos have the NFW (Navarro et al. 1996) density profile, $\rho \propto 1 / x(1+x)^{3}$, where $x=r / r_{s}$ is the radius in units of the break radius $r_{s}$. Each halo is characterized by its virial mass $M_{v i r}$ and the concentration $c=r_{v i r} / r_{s}$. The virial mass is defined by the radius $r_{v i r}$ at which the enclosed density exceeds the critical density by $\Delta_{c}(z)$. We estimated the concentration by the mean relation $c \simeq 9(1+z)^{-1}\left(M_{v i r} / 8 \times 10^{12} M_{\odot}\right)^{-0.14}$ from Bullock et al. (2000). Finally, the halo is assumed to have angular momentum $J$ specified by its spin parameter $\lambda=J|E|^{1 / 2} / G M_{\text {vir }}^{5 / 2}$ where the binding energy $|E|$ is computed using the virial theorem. 
We model galaxies as exponential disks with masses of $M_{d}=m_{d} M_{v i r}$ and scale lengths $r_{d}$. The disk is assumed to have angular momentum $J_{d}=j_{d} J$ and this is used to determine the disk scale length (see Mo et al. 1998). Unlike Mo et al. (1998) we added a bulge modeled as a Hernquist (1990) profile with mass $M_{b}=m_{b} M_{v i r}$ and an empirically estimated scale length of $0.045 r_{d}$ from the photometry of galaxies. We usually assumed that the total specific angular momentum of the baryons was the same as that of the dark matter but that all the angular momentum is in the disk component (i.e. $j_{d}=m_{d}+m_{b}$ and $\left.j_{b}=0\right)$. We use the standard Blumenthal et al. (1986) model for the adiabatic compression of the dark matter by the cooled baryons.

Finally, we used a simplified version of the Cole et al. (2000) cooling model from their semi-analytic models. We are interested in cooling because it plays an important role in determining the boundary between galaxies (where the gas can cool and form stars) and groups/clusters (where it remains hot). The Cole et al. (2000) model provides an estimate of the cooling time as a function of radius in a halo of a given virial temperature (i.e. mass), $\tau_{\text {cool }}(M, r)$, and we determine the cooled baryonic mass fraction $f_{\text {cool }}(M, z)$ at redshift $z$ by the mass fraction inside the radius where the cooling time equals the current age, $t(z)-t_{\text {form }}(M, z)=\tau_{\text {cool }}\left(M, r_{\text {cool }}\right)$. If the global baryon fraction is $\left(m_{d}+m_{b}\right)_{0}$ then we model the halo by an adiabatic compression model of cold baryon mass fraction $m_{d}+m_{b}=\left(m_{d}+m_{b}\right)_{0} f_{\text {cool }}(M, z)$. Assuming halos start as fair samples of the universe, the global baryon fraction is $\left(m_{d}+m_{b}\right)_{0}=\Omega_{b} / \Omega_{0}=0.13$. However, the final cold baryon fraction can be smaller, $\Omega_{b, \text { cool }} \leqslant \Omega_{b}$, because star formation and feedback can reheat the baryons which initially cooled (see SA).

For our scalings, the peak rotation velocity of an NFW halo of mass $M$ is

$$
v_{m o d, 0}(M)=186\left(M / 10^{12} M_{\odot}\right)^{0.30} \mathrm{~km} \mathrm{~s}^{-1}
$$

which rises to

$$
v_{\text {mod }}\left(M, m_{d}, \lambda\right)=v_{\text {mod }, 0}(M)\left[1+\frac{314 m_{d}^{2} \Lambda^{-0.98}}{1+42.3 m_{d} \Lambda^{0.30}}\right]
$$

for a halo with cold baryon fraction $m_{d}$ (disk only, $m_{b}=0$ ) and an effective spin parameter of $\Lambda=\left(j_{d} / m_{d}\right)(\lambda / \bar{\lambda})$ normalized by a mean spin parameter of $\bar{\lambda}=0.05$ and valid for $0 \leqslant m_{d} \leqslant 0.15$ and $0.02 \leqslant \lambda \leqslant 0.1$ (see Mo et al. 1998 for the effects of varying $c$ ).

\section{Why Don't Cluster Lenses Exist?}

One of the most striking features of surveys for gravitational lenses is that cluster lenses do not exist. This statement may seem peculiar given the enormous attention devoted to lensing by rich clusters (e.g. Tyson in these proceedings), but it is simply another facet of the fact that rich clusters loom large in our imaginations despite being exponentially rare and containing a negligible galaxy or mass fraction. The known rich cluster lenses were all found by first finding a rich cluster and then searching for lensed sources behind them.

Only surveys which examine sources to see if they are lensed probe the halo mass function, because there is a mapping between the image separation distribution $d n / d \Delta \theta$ and the halo mass function $d n / d M$. For example, the CLASS survey (e.g. Browne \& Myers 2000, Philips et al. 2000) for lensed flat-spectrum radio sources has a nearly uniform selection function from $00^{\prime \prime} 3 \lesssim \Delta \theta \lesssim 15^{\prime \prime} .0$ and has found 18 lenses all with separations $\Delta \theta<3{ }^{\prime \prime}$. 0 . If we consider all 27 radio-selected lenses, there are two wider separation lenses (MG2016+112 and Q0957+561) reaching to $\Delta \theta \lesssim 66^{\prime \prime} 0$. Despite having the sensitivity to wide separations needed to find cluster lenses, the distribution is overwhelmingly domi- 


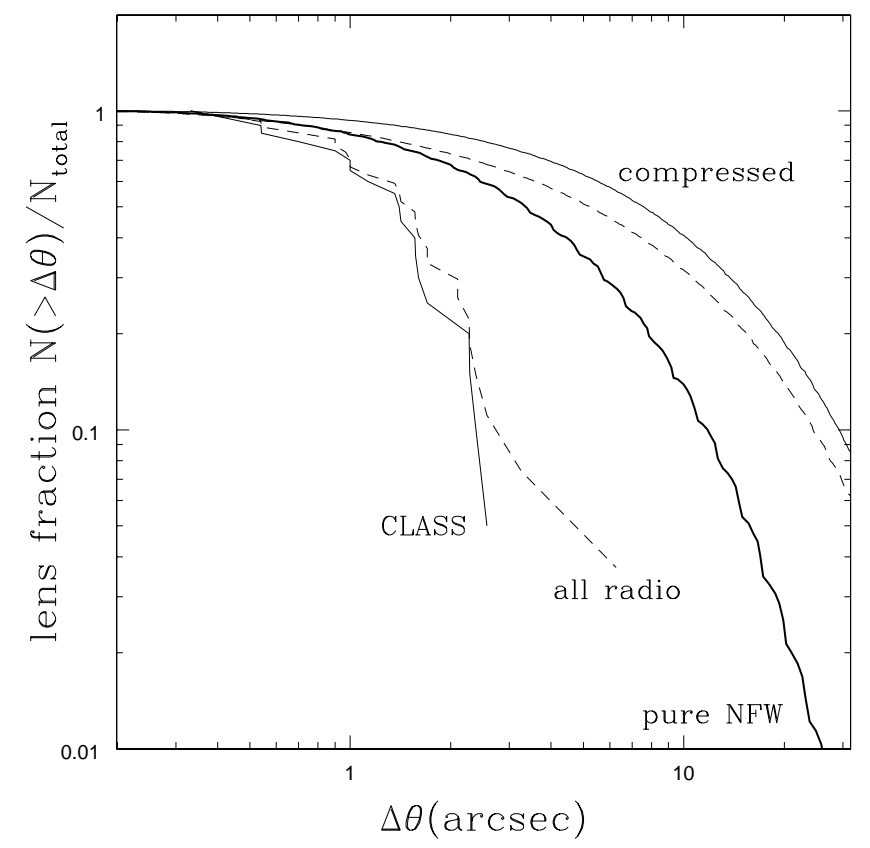

FiguRE 1. Predicted separation distributions without a cooling scale. The curves show the fraction of lenses with separations exceeding $\Delta \theta, N(>\Delta \theta) / N_{\text {total }}$. The observed distributions are shown by the curves labeled CLASS (for the CLASS survey lenses) and all radio (for all radio-selected lenses). The heavy solid line shows the distribution predicted by pure NFW models while the light solid (dashed) lines shows the distributions predicted by the adiabatic compression models with no bulge (a 10\% baryonic mass fraction bulge).

nated by galaxy lenses (average separations of $1{ }^{\prime \prime} .5$ ) with a few lenses due to groups and poor clusters on larger scales (see Fig. 1).

All calculations of the separation distribution of lenses combining the halo mass function with a model density distribution for the halos catastrophically fail to explain the observed distribution of image separations (e.g. Narayan \& White 1988, Kochanek 1995, Wambsganss et al. 1995, 1998, Maoz et al. 1997, Keeton 1998, Mortlock \& Webster 2000, Li \& Ostriker 2000, Keeton \& Madau 2000, Wyithe et al. 2000). Models normalized by the abundance of rich clusters correctly find that rich cluster lenses are rare, but then grossly under predict the number of galaxy-scale lenses (see Fig. 1). A purely phenomenological approach based on the local properties of galaxies, by contrast, predicts the observed properties of the lenses well (e.g. Kochanek 1996, Keeton et al. 1998). These models have modest difficulty explaining the largest lenses found in systematic surveys $\left(\Delta \theta \simeq 6{ }^{\prime \prime} 0\right)$ and include no rich cluster lenses.

Keeton (1998), followed by Porciani \& Madau (2000) and Kochanek \& White (2001), demonstrated that the origin of the problem lay in neglecting the baryonic physics which makes the density structure of the lenses depend strongly on the mass scale. Any model based on the halo mass function which assumes that the density distributions of the halos vary smoothly and continuously with mass leads to predictions for the image separation distribution which catastrophically fail to match the data. Keeton (1998) demonstrated it for singular isothermal sphere (SIS) and NFW models, while Kochanek \& White (2001) demonstrated it for the adiabatically compressed models described in $\S 2$ and illustrated in Fig. 1. The key, which can be understood self-consistently based on the adiabatic com- 


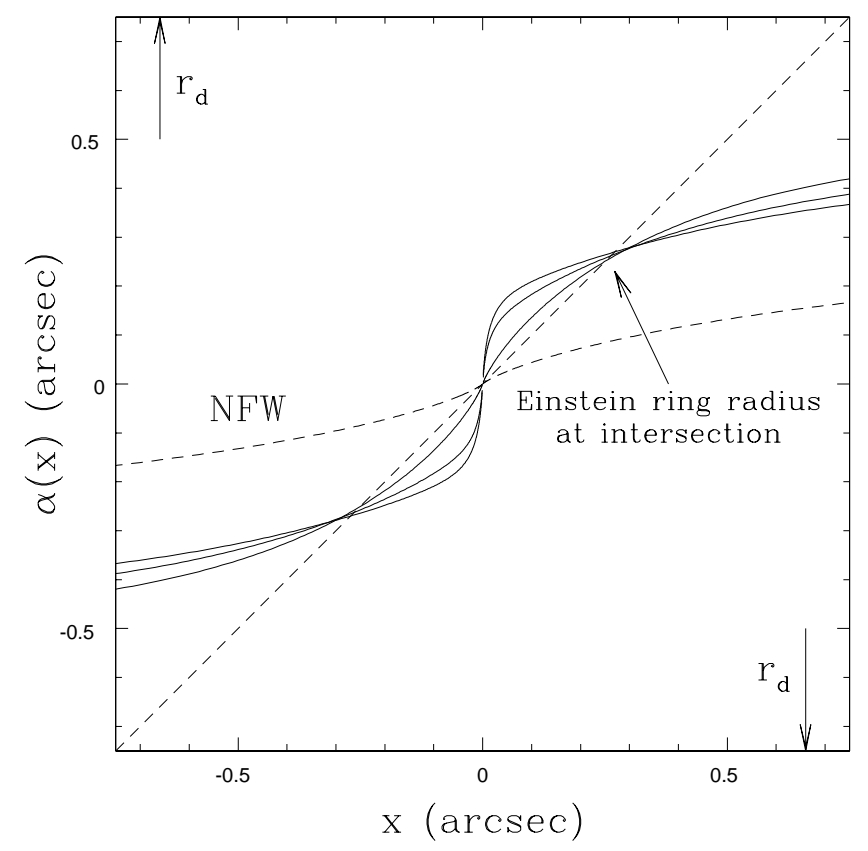

FIGURE 2 . The bending angles, $\alpha(x)$, produced by a $10^{12} M_{\odot}$ halo at $z_{l}=0.5$ with concentration $c=8$ for a source at $z_{s}=2.0$. The dashed curve shows the bend angle for the initial sub-critical NFW halo. The solid curves show the bend angles found after the baryons cool assuming a baryonic mass fraction of $m_{d}+m_{b}=0.05$, a spin parameter $\lambda=0.04$, and that the disk contains all the initial baryonic angular momentum, $j_{d}=0.05$. The three solid curves are for bulge-to-disk mass ratios $m_{b} / m_{d}=0$ (shallowest rise), 0.1 and 0.2 (steepest rise) respectively. The tangential critical line of the lens (the Einstein ring) is located at the point where the (dashed) $45^{\circ}$ line intersects the bending angle, and the radial critical line is located where a $45^{\circ}$ line is tangent to the bending angle. An arrow points to the location of the tangential critical line of the adiabatically compressed models. The arrows on either side of the figure indicate the (angular) disk scale length $r_{d}$ at the model redshift.

pression models described in $\S 2$ (Kochanek \& White 2001), is that the lensing efficiency of a halo increases dramatically as the halo is compressed by the cooling baryons. For example, Fig. 2 shows the change in the deflection profile (bend angle) for a $10^{12} M_{\odot}$ halo at $z_{l}=0.5$ with a source at $z_{s}=2$ between the initial NFW model and the final adiabatically compressed model with mass fraction $m_{d}+m_{b}=0.05$ in cold baryons. Where the initial NFW halo is sub-critical and unable to generate multiple images, the compressed halo is super-critical. The final cross section depends strongly on the details of the baryon distribution, as the models with bulge-to-disk mass ratio of $m_{b} / m_{d}=20 \%$ are significantly better lenses than those without a bulge component.

Keeton (1998) and Porciani \& Madau (2000) phenomenologically solved the problem of determining the global separation distribution by breaking the mass function into a high mass (cluster) distribution modeled by standard NFW profiles and a low mass (galaxy) distribution modeled using the local properties of galaxies rather than the theoretical mass function. In order to fit the observed separation distribution, the break had to be located at a cooling mass scale near $M_{c}=10^{13} M_{\odot}$. Given the difficulty in performing ab initio calculations for the final density structure of galaxies (as illustrated by the strong dependence of the bend angles on the bulge mass fraction in Fig. 2), such phenomeno- 


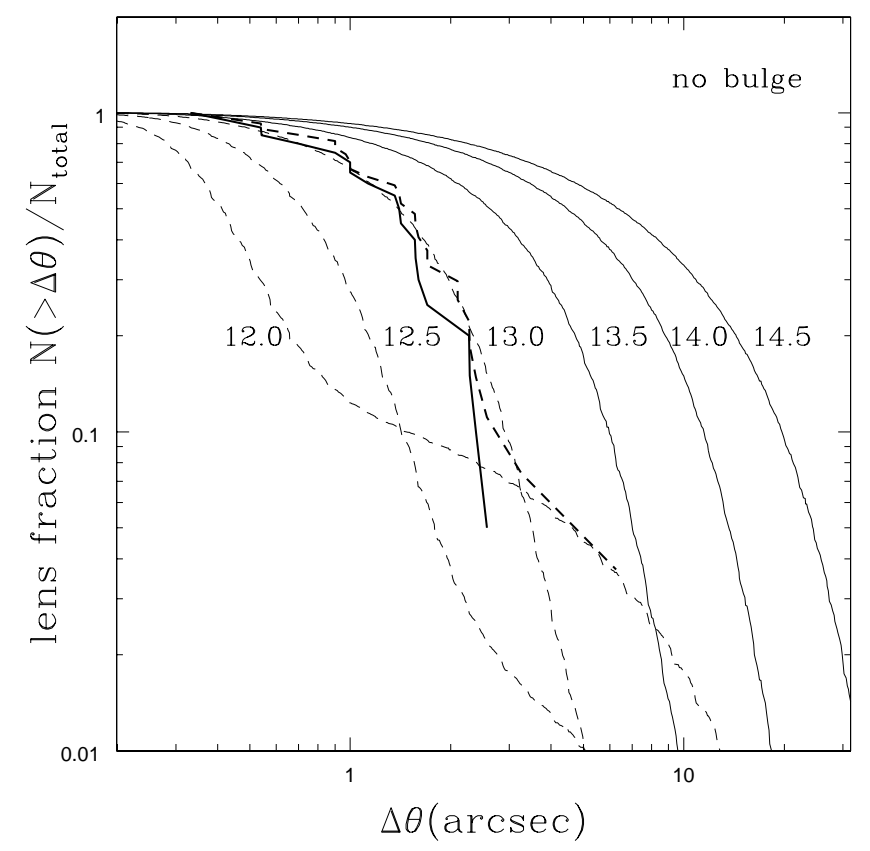

FiguRE 3. Predicted separation distributions with a cooling scale for models without a bulge and cold baryon fraction $m_{d}+m_{b}=0.05$ in halos below a cooling mass scale $M_{c}$. The curves are labeled by $\log M_{c} / M_{\odot}$. The heavy solid (dashed) curve shows the observed distribution of the CLASS (radio-selected) lenses.

logical methods are likely to be more quantitatively useful than current attempts at ab initio calculations.

While recognizing this limitation on the quantitative use of current theoretical models for the final density structure of galaxies, Kochanek \& White (2001) used the theoretical models to illustrate and isolate the physics needed to produce the observed separation distribution from the halo mass function. They first assumed that for halos less massive than a cooling mass scale $M_{c}$, baryonic mass fraction $m_{d}+m_{b}=0.05$ of the halos cools. Fig. 3 shows the predicted separation distributions as a function $M_{c}$. Recall, from Fig. 1, that if either all (high $M_{c}$ ) or no (low $M_{c}$ ) halos cool, then we cannot match the observations. Only for a cooling scale $M_{c} \sim 10^{13} M_{\odot}$ can we produce a sharp break in the separation distribution on the $\Delta \theta=33^{\prime \prime} 0$ scale of the observations. The exact scale depends on the density structure of the cooled halos, with $M_{c}$ decreasing from $10^{13} M_{\odot}$ to $5 \times 10^{12} M_{\odot}$ when we add a $m_{b} / m_{d}=0.1$ bulge. Interestingly, cosmological hydrodynamic simulations also find that the cooled baryon fraction reaches $50 \%$ on mass scales near $10^{13} M_{\odot}$ (e.g. Pearce et al. 1999). The required cooling mass scale also depends on the cold baryon fraction, with $M_{c}$ decreasing as $\log _{10} M_{c} / M_{\odot} \simeq 13.6-\left(m_{d}+m_{b}\right) / 0.15$ when the cold baryon fraction $m_{d}+m_{b}$ increases. The Kolmogorov-Smirnov (K-S) test probability of fitting the observed separation distribution is shown in Fig. 4 for a range of cold baryon fractions.

These two parameters, the cooling mass scale and the cold baryon fraction, are not independent. The cooling mass scale $M_{c}$ at any epoch is the mass scale where the cooling time is roughly equal to the average age of the halos. Fig. 4 also shows that for the current epoch, the two time scales are equal near $M_{c}=10^{13} M_{\odot}$ for $m_{d}+m_{b}=0.05$. 


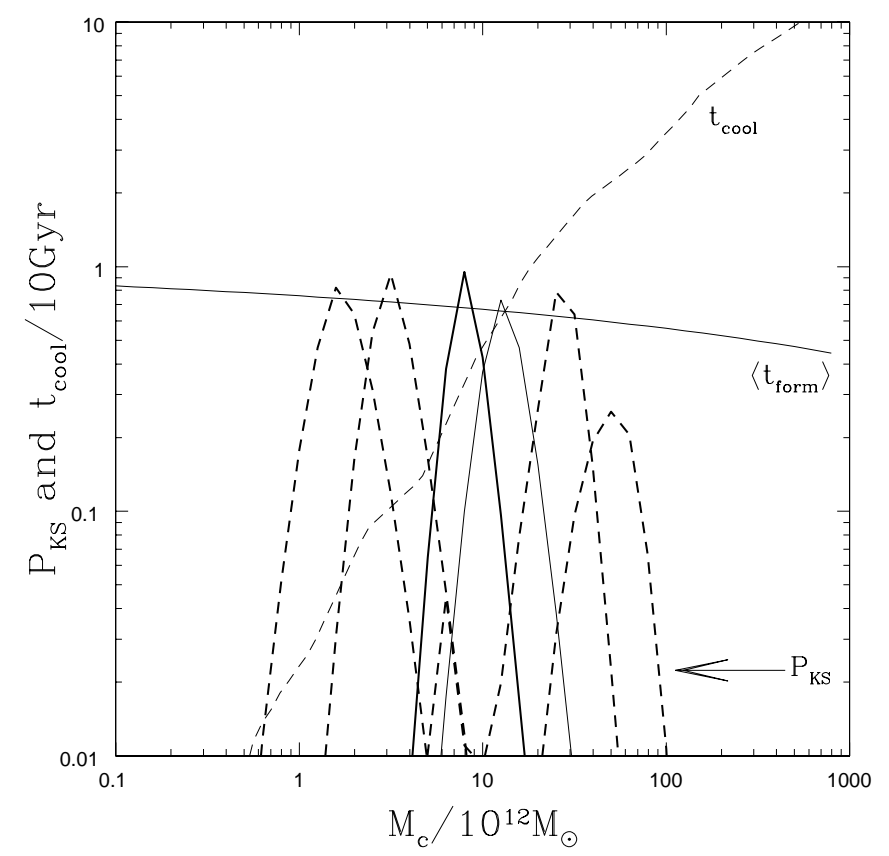

Figure 4. The Kolmogorov-Smirnov probability, $P_{K S}$, of fitting the observed separation distribution of CLASS lenses as a function of the cooling mass scale $M_{c}$. The heavy (light) solid curves indicated by the arrow show the K-S probability for models with $m_{b}+m_{d}=0.05$ without (with) a $m_{b} / m_{d}=0.10$ bulge. The heavy dashed curves show the K-S probabilities for models with lower $\left(m_{b}+m_{d}=0.01\right.$ and 0.02$)$ or higher $\left(m_{b}+m_{d}=0.10\right.$ and 0.20$)$ baryon fractions where the optimal cooling mass decreases as the baryon fraction rises. The light dashed curves show the cooling time in units of 10 Gyr for the radii enclosing $50 \%$ of the baryonic mass for the standard model. The light solid line shows the time since the average formation epoch $\left(\left\langle t_{\text {form }}\right\rangle\right)$ in units of 10 Gyr assuming $h=0.67$.

A lower cold baryon fraction requires a mass scale $M_{c}$ for which there is insufficient time for the baryons to cool. A higher cold baryon fraction requires mass scales where there is too much time for the baryons to cool. Since (to first order!) cooling physics combined with the baryonic mass fraction determines $M_{c}$, the fundamental physical parameter leading to the observed separation distribution is the cosmological baryon density $\Omega_{b}$. If all baryons cooled and remained cold, then there would be no ambiguities to this statement. However, star formation and feedback can reheat large fractions of the baryons even if they cool initially (see SA), so that the cosmological density in cold baryons, $\Omega_{b, \text { cool }}$, can be significantly less than the total density in baryons $\Omega_{b}$. In our simple models we assume that halos start with (eventually cold) baryon mass fraction $\left(m_{d}+m_{b}\right)_{0}=\Omega_{b, \text { cool }} / \Omega_{m} \leqslant \Omega_{b} / \Omega_{m}=0.13$ and that the fraction which has cooled and compressed a halo of mass $M$ at redshift $z$ is $m_{d}+m_{b}=\left(m_{d}+m_{b}\right)_{0} f_{\text {cool }}(M, z)$ where the simple model of the cooling function outlined in $\S 2$ determines $f_{\text {cool }}(M, z)$. Fortunately, the estimates of $\Omega_{b, \text { cool }}$ depend very weakly on changes in $f_{c o o l}$, the lens sample used, or the addition of a small bulge component.

The $\mathrm{K}-\mathrm{S}$ test probability of fitting the observed separation distribution as a function of the cold baryon density $\Omega_{b \text {,cool }}$ is shown in Fig. 5 . With little sensitivity to the details, models with $0.015 \lesssim \Omega_{b \text {,cool }} \lesssim 0.025$ agree with the data. While the preferred range is less than the total baryon density $\Omega_{b}=0.04$ in the input cosmology, it significantly 


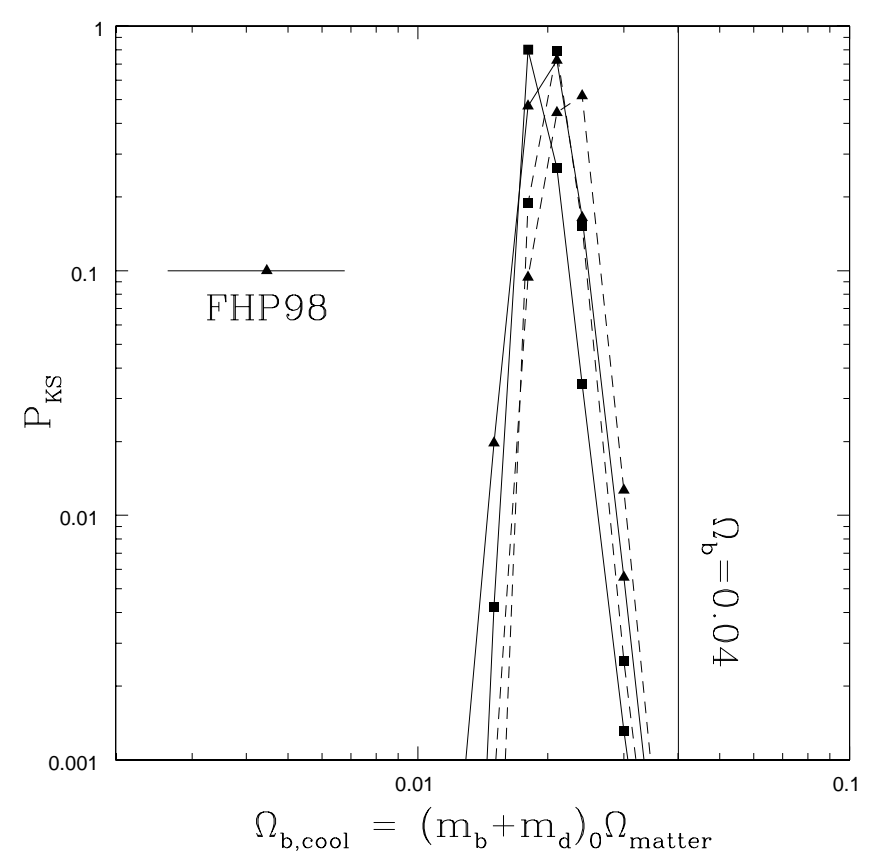

FIGURE 5. Kolmogorov-Smirnov test probability of fitting the separation distribution of CLASS lenses as a function of $\Omega_{b, \text { cool }}$. The squares (triangles) indicate models with no bulge (with a $m_{b} / m_{d}=0.1$ bulge), and the solid (dashed) lines correspond to fitting the CLASS lenses (all radio lenses). The point with horizontal error bar is the estimate by Fukugita, Hogan \& Peebles 1998 for the cold baryon (stars, remnants, cold gas) content of galaxies. The vertical line marks the total baryon content in the concordance model.

exceeds the estimates of $0.0045 \lesssim \Omega_{b \text {,cool }} \lesssim 0.0068$ for the cold baryon fraction (stars, cold gas and stellar remnants) in local galaxies by Fukugita, Hogan \& Peebles (1998). This is a weighted average over all galaxies rather than simply that of massive galaxies, but the conflict is probably present even for massive galaxies like the Milky Way. Models of the Galaxy by Dehnen \& Binney (1998) find a baryonic mass fraction $m_{d}+m_{b} \simeq$ 0.08 corresponding to $\Omega_{b, \text { cool }} \simeq 0.024$ at $100 \mathrm{kpc}$. As we increase the radius we include additional dark matter but no new baryons, so the baryon fraction drops to $0.008 \lesssim$ $\Omega_{b, \text { cool }} \lesssim 0.012$ for a halo with an outer extent of $200-300 \mathrm{kpc}$. While the difference is smaller, it suggests the existence of a dynamical baryon discrepancy, in which the cold baryon fraction required to explain the observed dynamical properties of galaxies exceeds standard accountings for the cold baryons in galaxies.

\section{The Local Velocity Function}

The distribution of gravitational lens image separations is the cleanest dynamical probe of the halo mass function because the image separation is directly related to the underlying halo density distribution and because lensing selects halos without any dependence on the luminosity of the baryons. Unfortunately, while the number of known lenses is growing relatively rapidly, the overall size of the sample is limited. Our alternative dynamical probe of the mass function is the local velocity function of galaxies and clusters. The local velocity function has the opposite problems from the lenses, with far lower statistical uncertainties but far higher systematic uncertainties. First, selection meth- 
ods for galaxies and clusters depend on the luminosity and surface brightness of the halo. Second, the selection methods for galaxies and groups/clusters are inhomogeneous, leading to significant systematic difficulties when assembling a global velocity function incorporating both. Third, local dynamical probes of galaxies and clusters have many more ambiguities than gravitational lens image separations because they are only indirectly related to the mass distribution. This problem is worst for galaxies where there are significant systematic uncertainties in the relationship between stellar kinematics and the underlying mass distribution.

Most estimates of the local velocity function of galaxies have been made because it is an essential element in estimates of gravitational lens cross sections, separation distributions, and related statistics (e.g. Turner et al. 1984, Fukugita \& Turner 1991, Kochanek 1993, 1996; Maoz \& Rix 1993; Falco et al. 1998; Helbig et al. 1999). Cole \& Kaiser (1989) pointed out that the velocity function can also be used to test models of galaxy formation, the power spectrum and cosmology directly. This motivated estimates of the local velocity function by Shimasaku (1993) and Gonzalez et al. (2000) and further theoretical investigations by Sigad et al. (2000), Newman \& Davis (2000), and Bullock et al. (2001). All derivations of the galaxy velocity function use the Faber-Jackson (1976) and Tully-Fisher (1977) relations between luminosity and velocity to perform a variable transformation from a locally measured luminosity function into the velocity function. Far more effort has focused on determining the mass or velocity (temperature) function of groups and clusters (see the reviews by Rosati \& Donahue in these proceedings) and given the greater familiarity of these results we will not review them in any detail. It is difficult to merge the two, to produce a global view of the mass function, because of the problems in obtaining complete, well-understood samples of groups and then determining their masses or velocities (e.g. Mahdavi et al. 2000).

Existing observational estimates of the velocity function of galaxies suffer from (at least!) five systematic problems. First, deriving a velocity function requires the luminosity function of galaxies by type since the kinematics of pressure-supported early-type and rotation-supported late-type galaxies are very different. Unfortunately, there are large systematic differences between morphologically-typed and spectrally-typed luminosity functions. However, in Kochanek et al. (2001b) we find clear evidence that current spectrally-typed luminosity functions have internal inconsistencies which make the morphologically-typed surveys the better option at present. Second, the luminosity functions and the kinematic relations used to construct the velocity function are derived on different magnitude scales, leading to systematic shifts in the velocity scale. Third, by separating the derivations of the luminosity function and the kinematic relations, covariances which are important to the uncertainties in the velocity function are lost. Fourth, the classification method used for the luminosity function (morphological or spectral) is not the same as the kinematic classification (pressure or rotation supported) used to define the kinematic relations. The galaxies found in kinematic samples do not even represent fair samplings of the classifications used to construct the luminosity function as they are dominated by $\mathrm{E}$ and $\mathrm{Sb} / \mathrm{Sc}$ galaxies with few S0/Sa galaxies. Fifth, the kinematic velocities have specific observational definitions that are not easily translated into theoretically calculated quantities. For example, the central stellar velocity dispersion of an early-type galaxy is non-trivially related to the peak circular velocity of a model halo.

In Pahre et al. (2001) we took three steps to reducing these systematic uncertainties. First, we started from a large, local infrared luminosity function (Kochanek et al. 2001a) derived from the 2MASS (Skrutskie et al. 1997) survey. The galaxies were morphologically classified and the classifications are internally consistent and consistent with other local, morphologically classified samples (Kochanek et al. 2001b). We then matched galaxies 


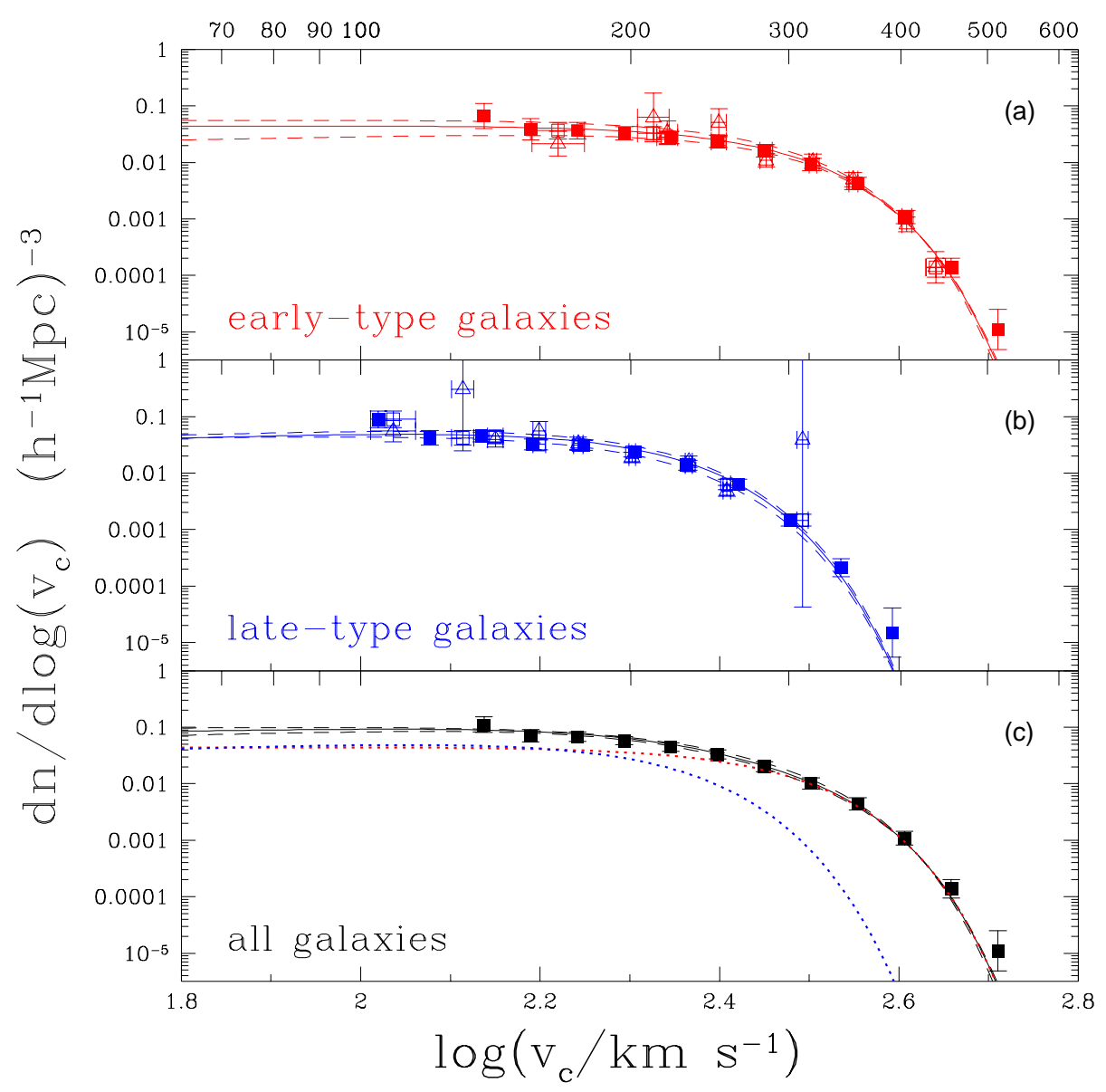

Figure 6. (a) The early-type galaxy velocity function. The curves show our parametric estimates of the velocity function. The solid curve is our standard estimate with the galaxy type boundary at $T=-0.5$, while the upper (lower) curves show that the number of low velocity early-type galaxies is strongly affected by shifts in the type boundary to $T=+0.5(-1.5)$. The points show the three different non-parametric estimates of the velocity function derived from the binned, non-parametric estimate of the luminosity function. The solid squares use the analytic Faber-Jackson relation to transform both the magnitude and the density, the open squares use it only to transform the density, and the open triangles do not use it at all. (b) The late-type galaxy velocity function. The curves and symbols are the same as in (a). (c) The total galaxy velocity function. The curves are the same as in (a) and (b), but now calculated for all galaxies simultaneously. The individual early- and late-type galaxy contributions to the velocity function are plotted as dotted lines.

in the 2MASS sample to several modern kinematic surveys to construct Faber-Jackson and Tully-Fisher relations in the same magnitude system as was used to derive the luminosity function. We then combined the luminosity function and the kinematic relations including the full variable covariances of the functions to determine the velocity function. We also explored non-parametric models for the velocity function where we minimized the use of functional forms for the luminosity function or the kinematic relations in favor of the raw, binned data. Fig. 6 shows the resulting velocity functions, and Fig. 7 compares our velocity function to other derivations (from Gonzalez et al. 2000) and to 


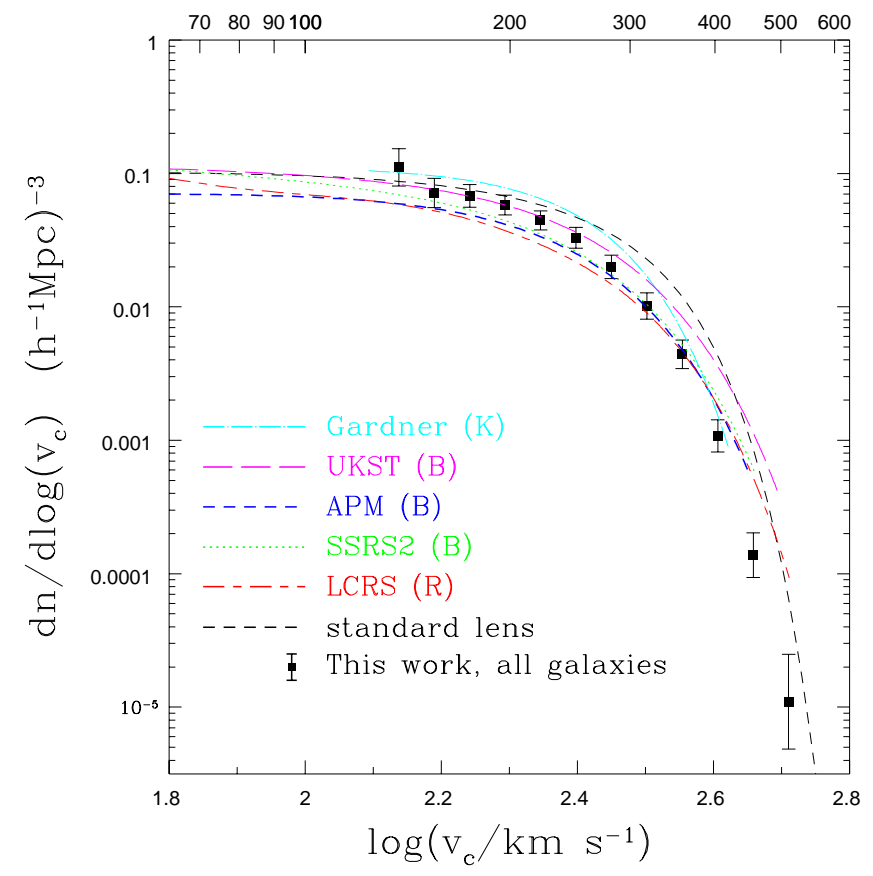

FiguRE 7. The total galaxy velocity function compared to previous estimates from Gonzalez et al. (2000) and Falco et al. (1998). The standard lens model can be offset in velocity because of differences in the observational method (see $\S 5$ ).

the standard result from gravitational lens statistics (Falco et al. 1998). While our result has reduced systematic errors compared to earlier derivations, we have not eliminated problems created by the difference between morphological and kinematic types or the problems in relating observed and theoretical velocities.

Our theoretical model, tuned to reproduce the distribution of gravitational lens separations in $\S 3$, also estimates the peak circular velocity as a function of mass, as shown in Fig. 8. Below the cooling mass scale, the baryons compress the halos and shift the mass-velocity relation $v_{c}(M)$ upwards. The amount of the shift depends on both the assumed baryon distribution (as illustrated by comparing models with bulge-to-disk mass ratios of $m_{b} / m_{d}=0$ and 0.1 ) and redshift. With added physics (such as the reheating mechanisms in the SA models), the simple power-law structure below the cooling mass scale changes. We can use the mass-velocity relation to derive the velocity function from the mass function as a simple variable transformation (eqn. 6.2 with $p_{g}(M)=1$ ) with the results shown in Fig. 9. We used three $v_{c}(M)$ relations: the virial velocity of the NFW halos, the peak circular velocity of the NFW halos, and the $v_{c}(M)$ relation produced by the model which best fit the gravitational lenses. We also extended the observed velocity function by adding a crude estimate for the contribution of groups and clusters to the local velocity function. We estimated the cluster contribution from the Blanchard et al. (2000) X-ray temperature function combined with the Wu et al. (1999) relation between the temperature and the velocity dispersion (a simple thermodynamic conversion is nearly identical) truncated to $T>0.5 \mathrm{keV}$. This produces the small kink in the estimate near $v_{c}=400 \mathrm{~km} \mathrm{~s}^{-1}$. On cluster scales the models and the data agree relatively well, although a modestly lower normalization for the mass function would fit the data better. On galaxy scales, the models without the compression by the baryons grossly un- 


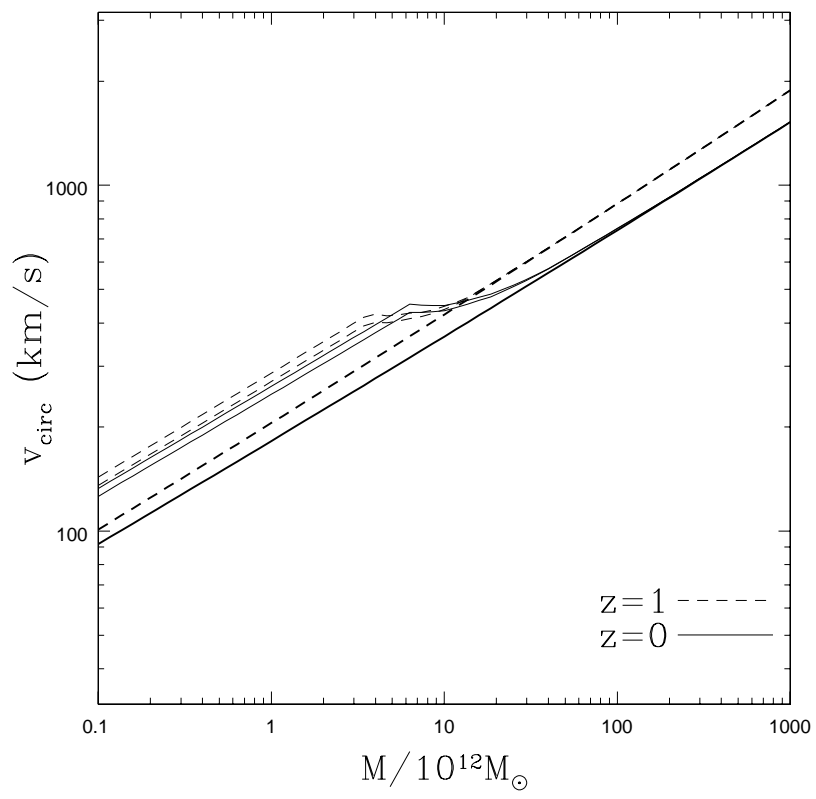

FiguRE 8. The global relation between mass and circular velocity at redshifts zero (solid) and unity (dashed). The heavy curves show the peak circular velocity of the NFW model. The light curves show the peak circular velocity including the baryonic cooling and adiabatic compression from the $\Omega_{b, \text { cool }}=0.018$ model. The upper light curve is the model with no bulge component $\left(m_{b} / m_{d}=0\right)$ and the lower light curve is the model with a bulge $\left(m_{b} / m_{d}=0.1\right)$. The bulge slightly reduces the peak rotation velocity (because it increases the angular momentum per unit mass of the disk) while making the rotation curve flatter.

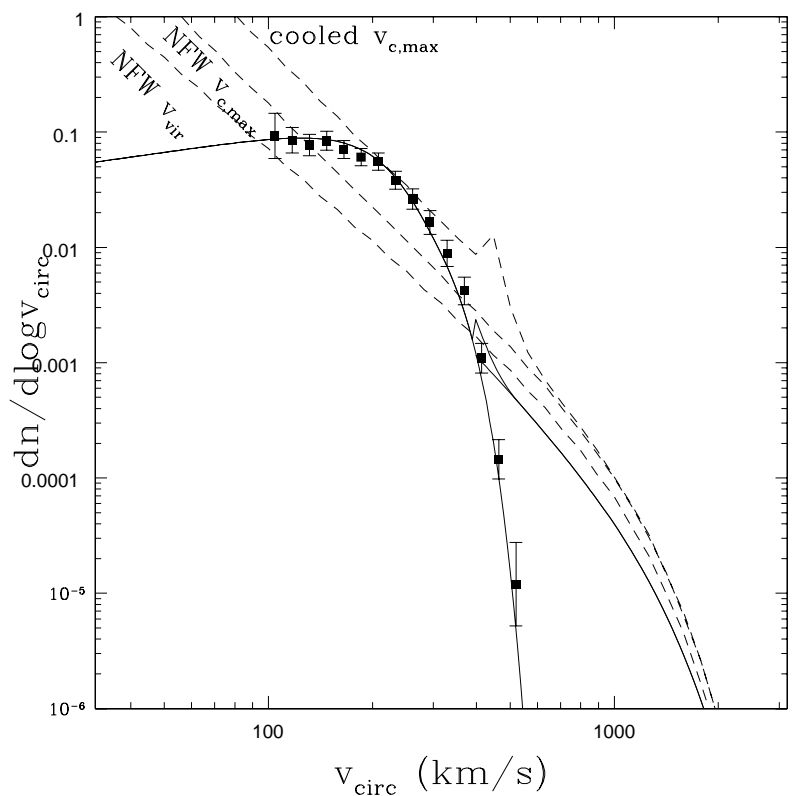

Figure 9. The velocity function $d n / d \log v_{c}=(d n / d \log M)\left|d \log M / d \log v_{c}\right|$. The solid curves show the local velocity function of galaxies (low $v_{\text {circ }}$ ) and clusters (high $v_{c}$ ) and their sum. The points are the non-parametric velocity function of galaxies. From bottom to top, the dashed curves show the velocity functions derived using $d n / d M$ and the NFW virial velocity (labeled NFW $v_{\text {vir }}$ ), the peak circular velocity of the NFW rotation curve (labeled NFW $v_{c, \max }$ ) and the peak circular velocity of the adiabatically compressed model (labeled cooled $v_{c, \max }$ ). We used the $\Omega_{b, \text { cool }}=0.018$ model with no bulge. 
der predict the density of halos with observed velocities $200 \mathrm{~km} \mathrm{~s}^{-1} \lesssim v_{c} \lesssim 400 \mathrm{~km} \mathrm{~s}^{-1}$ while the models with adiabatic compression match the density relatively well.

Our model clearly has problems at low velocity and at the juncture between galaxies and groups/clusters. These are not apparent in the models for the gravitational lenses because the lens cross sections combined with the angular selection functions strongly suppress the contributions from halos with $v_{c} \lesssim 150 \mathrm{~km} \mathrm{~s}^{-1}$, and because the separation distribution smoothes the velocity function by an average over lens redshift. The peak in the model distribution near $400 \mathrm{~km} \mathrm{~s}^{-1}$ is an artifact of the flat slope of the $v_{c}(M)$ curves (see Fig. 8). The $v_{c}(M)$ relation has to be multi-valued in this region with a diminishing probability of forming a compressed galaxy halo and a rising probability of forming an uncompressed group halo (we will explore this further in §6). Gonzalez et al. (2000), who made a similar comparison to the velocity function of galaxies based on the Somerville \& Primack (1999) semi-analytic models, had very similar problems. Even though they used a model where feedback from star formation varied the cold baryon fraction to maximize the compression at $v_{c} \sim 250 \mathrm{~km} \mathrm{~s}^{-1}$ and to reduce it for higher and lower velocity halos, their model velocity function more closely resembles our theoretical curves than the observations.

\section{Deriving the Velocity Function From the Lens Separations}

As a final check of the consistency of the model, the distribution of lens separations and the velocity function, we can estimate the velocity function directly from the distribution of lens separations so that the comparison does not depend on our theoretical model from $\S 2$. We will compare only the shapes of the velocity function and not the absolute normalization (number per comoving Mpc) since the normalization introduces the uncertainties in the absolute numbers of gravitational lenses found by a survey. We assume that lenses can be modeled as singular isothermal spheres (SIS), which is broadly consistent with both lensing and dynamical data on the relevant scales (see Cohn et al. 2001), so that the observed image separation is a simple function of the circular velocity $v_{c}$ and the lens-source/observer source distance ratio, $\Delta \theta=\Delta \theta_{0}\left(v_{c} / v_{0}\right)^{2} D_{L S} / D_{O S}$ where $\Delta \theta_{0}=4 \pi\left(v_{0} / c\right)^{2}$ sets an arbitrary velocity scale for the calculation. In any flat cosmology, the normalized image separation distribution is

$$
\frac{1}{\tau} \frac{d \tau}{d \Delta \theta}=\frac{\Delta \theta^{2} S(\Delta \theta)}{\Delta \theta_{0}}\left[\int_{0}^{1} x^{2} d x \int_{0}^{\infty} d v \Delta \theta^{2} S(\Delta \theta) \frac{d n}{d v}\right]^{-1} \int_{v_{\min }}^{\infty} d v \frac{v_{0}^{2}}{v^{2}} \frac{d n}{d v}\left(1-\frac{v_{0}^{2}}{v^{2}} \frac{\Delta \theta}{\Delta \theta_{0}}\right)^{2}
$$

where $v_{\text {min }}=v_{0}\left(\Delta \theta / \Delta \theta_{0}\right)^{1 / 2}, 0 \leqslant S(\Delta \theta) \leqslant 1$ is the survey selection function for finding a lens of separation $\Delta \theta, x=D_{O L} / D_{L S}$, and $d n / d v$ is the velocity function. We can nonparametrically determine the velocity function using a variant of the step-wise maximum likelihood (SWML) luminosity function estimation method of Efstathiou et al. (1988). We approximate $d n / d v$ by a series of bins with density $n_{i}$ and then maximize the likelihood of finding the observed lens separations while holding the number of lenses in the bin centered on $v_{c}=300 \mathrm{~km} / \mathrm{s}$ fixed to unity. The latter constraint corresponds to ignoring the absolute comoving density of the lenses and considering only the shape of the velocity function. Eqn. (5.1) holds for any flat cosmological model.

The results for three lens samples are shown in Fig. 10. Sample A consists of the 20 lenses found in surveys based $8 \mathrm{GHz}$ VLA A-array maps of flat-spectrum radio sources by the CLASS and PMN (Winn et al. 2000) surveys. Sample B, with 27 lenses, adds the remaining radio-selected lenses. Sample C, with 46 lenses, adds the optically-selected quasar lenses. The model for the angular selection function is adjusted for the angular 


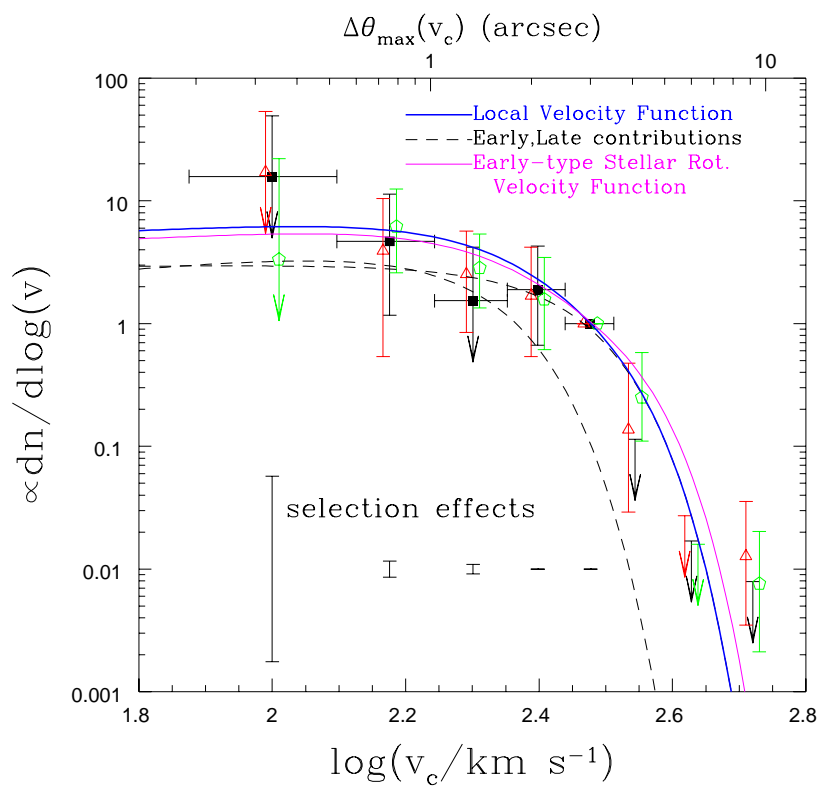

FIGURE 10. The velocity function estimated from gravitational lenses. The solid points are derived using only the flat-spectrum radio surveys, the triangles include the MIT-Greenbank survey, and the pentagons include quasar lenses. The triangles and pentagons are slightly offset in velocity to make them more visible. The horizontal error bars show the width of the velocity bins. The distributions are normalized by the logarithmic density at $v_{c}=300 \mathrm{~km} \mathrm{~s}^{-1}$. The solid (dashed) line shows our locally estimated total (early-type and late-type) velocity function. The error ranges labeled "selection effects" show the effects of plausible uncertainties in the angular selection function $S(\Delta \theta)$. The scale at the top of the figure shows the maximum image separation produced by a lens with circular velocity $v_{c}$. The mean separation is one-half the maximum.

sensitivity of the survey which found each lens. As we move from Sample A to C we trade increasing systematic uncertainties for decreasing statistical uncertainties, although we find that the derived velocity functions are mutually consistent given the statistical uncertainties. Only the lowest velocity bin, centered at $v_{c}=100 \mathrm{~km} / \mathrm{s}$ and corresponding to an average image separation of only $\langle\Delta \theta\rangle=0{ }^{\prime \prime} 15$, has a significant sensitivity to plausible errors in the models for the angular selection function. Be warned that the error bars in Fig. 10 are even more highly correlated than similar figures derived for luminosity functions of galaxies!

If we normalize our estimate of the velocity function of galaxies at the same velocity scale and superpose it on that of the lenses, the two distributions are remarkably similar (see Fig. 10) They have the same flat low-velocity slope as a function of $\log \left(v_{c}\right)$ and an exponential cutoff on the same velocity scale. Only in the highest velocity bin, whose density is driven by the need to produce the widest separation Q $0957+561$ lens with $\Delta \theta=6{ }^{\prime \prime} 1$, does the velocity function of the lenses show a clear deviation from that of galaxies even though the lenses represent the global velocity function rather than that of the galaxies alone. The small amplitude of the deviation is another illustration of the enormous impact of the baryons on the dynamical structure of halos which we discussed in $§ 3$. At low velocities the two distributions have the same flat slope, rather than the steeply rising slope of our estimates from the mass function. Note, however, that neither 
observational sample has significant data on velocity scales $v_{c} \lesssim 100 \mathrm{~km} \mathrm{~s}^{-1}$ where the deviations from the predictions begin to diverge rapidly.

\section{A Non-Parametric Description of the Velocity Function}

Our theoretical model is a gross oversimplification. It agrees with our two observational probes on the mass scales corresponding to massive galaxies and clusters, but has problems on the mass scales of groups and fails badly for low mass galaxies. These problems can be partially rectified by more sophisticated models (e.g. SA) which allow for more complicated variations in the cold baryon fraction with halo mass. In this section we outline a general, non-parametric approach to understanding the relationship between the mass function of halos and the velocity function of galaxies which we can use to characterize the problem without the complications of a full semi-analytic model.

Our starting point is that in all models, no matter their complexity, the halo mass function and the galaxy velocity function are related by the probability $P\left(v_{c} \mid M\right)$ that a halo of mass $M$ forms a detectable galaxy with circular velocity $v_{c}$,

$$
\frac{d n}{d v_{c}}=\int_{0}^{\infty} \frac{d n}{d M} P\left(v_{c} \mid M\right) d M .
$$

The conditional probability, which need not integrate to unity, includes the effects of all parameters governing the formation of galaxies such as the spin parameter $\lambda$, the collapse redshift, the halo merger history and its environment. If $P\left(v_{c} \mid M\right)$ is dominated by a sufficiently narrow ridge, so that it is reasonable to associate a characteristic velocity with halo mass, then we can approximate the integral (6.1) by

$$
\frac{d n}{d v_{c}}\left(v_{c}(M)\right)=p_{g}(M)\left|\frac{d v_{c}(M)}{d M}\right|^{-1} \frac{d n}{d M}
$$

where the unknown two-dimensional function is replaced by two one-dimensional functions with simple physical meanings. The first, $p_{g}(M)$, is the probability that a halo of mass $M$ forms a galaxy included in the velocity function, and the second, $v_{c}(M)$, is the circular velocity of the resulting galaxy. As a mathematical derivation we must assume that the fractional spread in velocity at fixed mass, $\sigma_{v}(M) / v_{c}(M)$, is small. Since the equivalent fraction at fixed luminosity is indeed small, and changing from mass to luminosity presumably raises rather than lowers the dispersion in galaxy properties, it seems likely that the expansion is justified. This model differs from that used in $\S 3$ and $\S 4$ where $p_{g}(M) \equiv 1$. Its main drawback is that it neglects "halos-in-halos" or the halo multiplicity function (e.g. Peacock \& Smith 2000, Soccimarro et al. 2001), although this should be a modest perturbation in the accounting for galactic halos $(\sim 10 \%$ in the numerical simulations of White et al. (2001). These two functions are implicitly included in semi-analytic models. For example, Gonzalez et al. (2000) fit the cold baryon fraction in the Somerville \& Primack (1999) models by $m_{d}(x)=0.1(x-0.25) /\left(1+x^{2}\right)$ for $x=v_{\text {mod }, 0}(M) / 200 \mathrm{~km} \mathrm{~s}^{-1}>0.25$ (see eqn. 2.1) to estimate $v_{c}(M)$ for the model. The velocity function predicted by this model cannot, however, reproduce the local velocity function of galaxies, as discussed in $\S 4$ (see Fig. 9).

Our decomposition of the problem into the formation probability $p_{g}(M)$ and the massvelocity relation $v_{c}(M)$ allows us to explore the problem in a model independent fashion. Unfortunately, the solution is not unique because we must determine both $p_{g}(M)$ and $v_{c}(M)$ from only one function. Fortunately, the two unknown functions should obey several constraints. First, if the normalization of the mass function is correct, $0 \leqslant p_{g}(M) \leqslant 1$. We can have $p_{g}(M)>1$ only if we interpret it as a normalization 


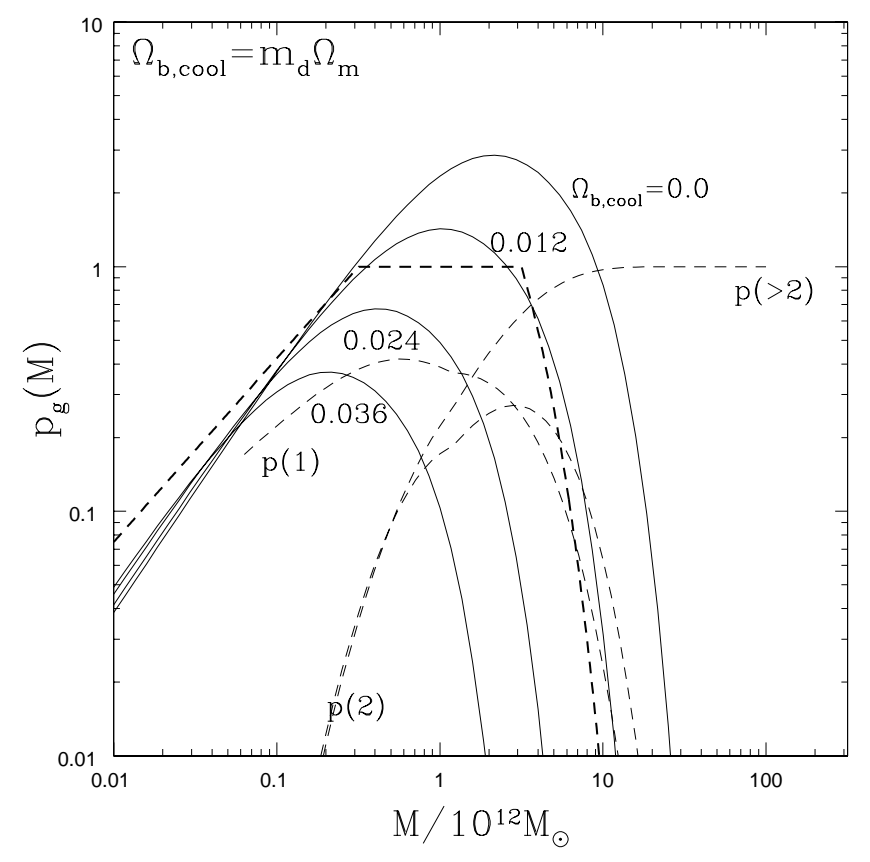

FiguRE 11. The galaxy formation probability, $p_{g}(M)$, required for models with constant cold baryon fractions. The curves are labeled by their cold baryon density, $\Omega_{b, \text { cool }}=m_{d} \Omega_{m}$. The upper curve is for halos with no cooled baryons $\left(\Omega_{b, \text { cool }}=0\right)$ and the lowest curve is for halos with $\Omega_{b \text {, cool }} \simeq \Omega_{b}$. The heavy dashed curve is the standard model from our attempt to non-parametrically adjust $v_{c}(M)$ in order to maximize the mass range over which $p_{g}(M)=1$. The light dashed curves show the probability of forming one $(p(1))$, two $(p(2))$ or at least two $(p(>2))$ galaxies as a function of halo mass in one of the halo multiplicity models of Scoccimarro et al. (2001). The Scoccimarro et al. (2001) models are low because they underestimate the comoving density of galaxies (see text).

error in the mass function or if the halo multiplicity function on galactic mass scales differs significantly from unity. Second, cooling baryons should only increase the halo circular velocity, $v_{c}(M) \geqslant v_{\text {mod }, 0}(M)$. Third, the compression must be bounded by models in which all the available baryons have cooled, $v_{c}(M) \leqslant v_{\text {mod }}\left(M, m_{d}=\Omega_{b} / \Omega_{m}, \lambda\right)$. Note that this upper bound is more model dependent than the lower bound.

Suppose that the standard adiabatic compression models are correct and that the dimensionless parameters (cold baryon fraction, spin parameter ...) are the same for all galaxies. These parameters fix $v_{c}(M)$, allowing us to calculate the galaxy formation probability required to produce the galaxy velocity function from the halo mass function. Fig. 11 shows the implied $p_{g}(M)$ for various cold baryon fractions $\left(m_{d}=0,0.04,0.08\right.$ and 0.12) and a fixed spin parameter $(\lambda=\bar{\lambda})$. At low mass, $p_{g}(M) \sim M$ is needed to match the steep slope of the mass function to the shallow slope of the velocity function, and at high mass it has the exponential cutoff cutoff of the velocity function. The peak probability and the corresponding mass scale decrease systematically as we raise the cold baryon fraction. If the compression is too small $\left(m_{d} \lesssim 0.04\right.$ or $\left.\Omega_{b, \text { cool }} \lesssim 0.01\right)$, we must increase the normalization of the halo mass function (i.e. the power spectrum) in order to avoid a region with $p_{g}(M)>1$. When the compression of the halos is large ( $m_{d} \gtrsim 0.08$ or $\Omega_{b, \text { cool }} \gtrsim 0.02$ ), either the velocity function is incomplete or we must lower the normalization of the mass function. 


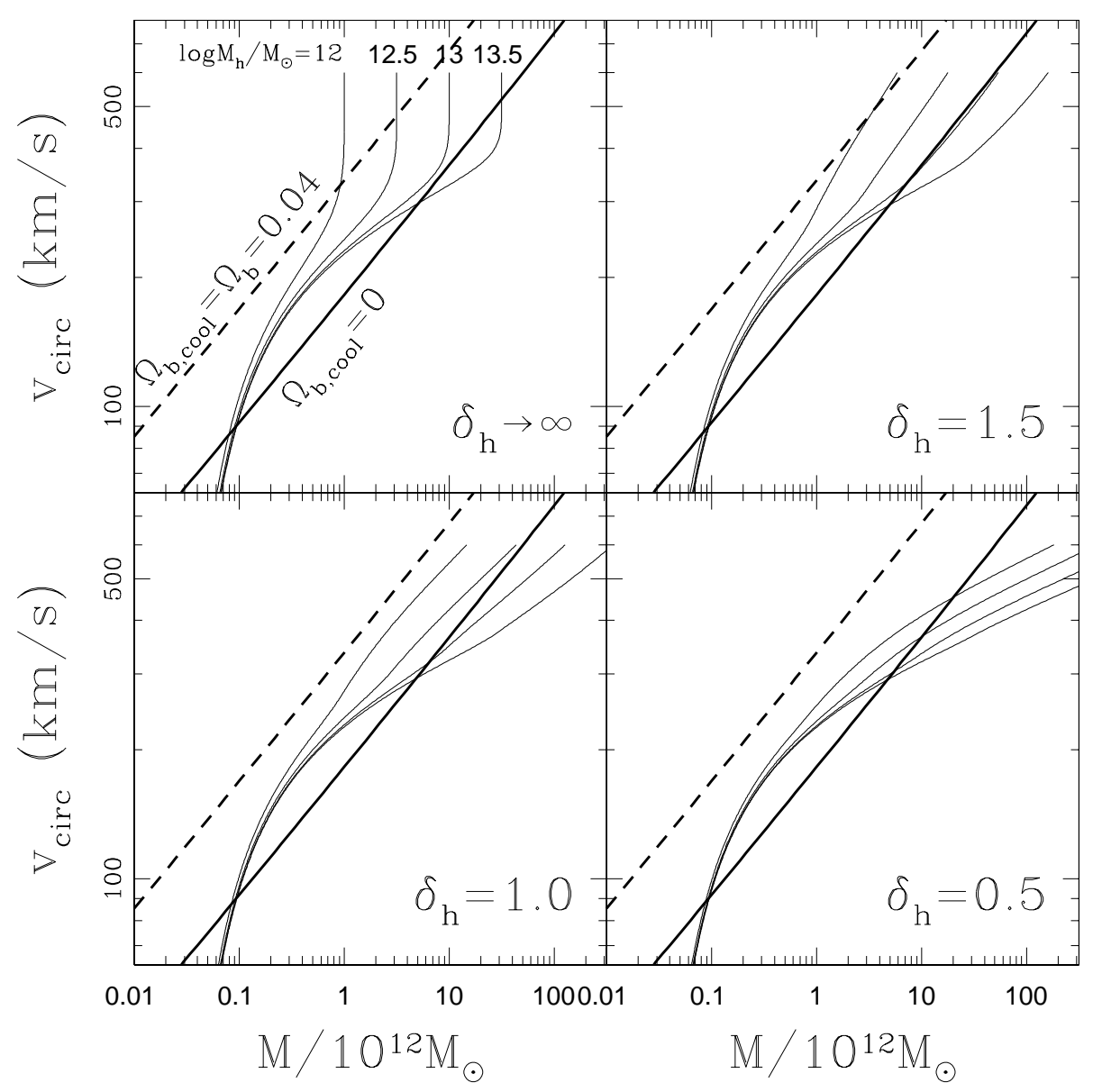

Figure 12. The effect of the high mass cutoff in the galaxy formation probability on the mass-velocity relation. The four light curves in each panel show the mass-velocity relations $v_{c}(M)$ needed to produce the observed velocity function given high mass cutoffs in the formation probability of $\log M_{h} / M_{\odot}=12.0,12.5,13.0$ and 13.5 (from left to right). The different panels show the effect of changing the cutoff exponent, with values of $\delta_{h} \rightarrow \infty$ (top left), 1.5 (top right), 1.0 (lower left) and 0.5 (lower right) covering the range from an infinitely sharp cutoff to a fairly shallow cutoff. The heavy curves show the permitted range for $v_{c}(M)$ with a lower boundary set by the circular velocity of uncompressed halos (the heavy solid line) and the upper boundary set by the circular velocity of halos in which all the available baryons have cooled (the heavy dashed line) The upper bound, which assumes $\lambda=\bar{\lambda}=0.05$ and $j_{d}=m_{d}$, is significantly more model dependent than the lower bound.

While these models clearly simplify the structure of the mass-velocity relation $v_{c}(M)$ by using models with fixed dimensionless parameters, they do not greatly exaggerate the dominant role of the formation probability in producing the observed shape of the velocity function. The requirement that a physical mass-velocity relation be bounded by the zero compression and maximal compression models prevents us from shaping $v_{c}(M)$ to produce the velocity function with the formation probability held constant over much more than one decade in mass. We can illustrate this by using a parametric model for $p_{g}(M)$ with a constant formation probability $p_{g}=p_{0}$ between $M_{l}<M<M_{h}$, a powerlaw $p_{g}=p_{0}\left(M / M_{l}\right)^{\delta_{l}}$ below $M_{l}$, and an exponential $p_{g}=p_{0} \exp \left(1-\left(M / M_{h}\right)^{\delta_{h}}\right)$ above 


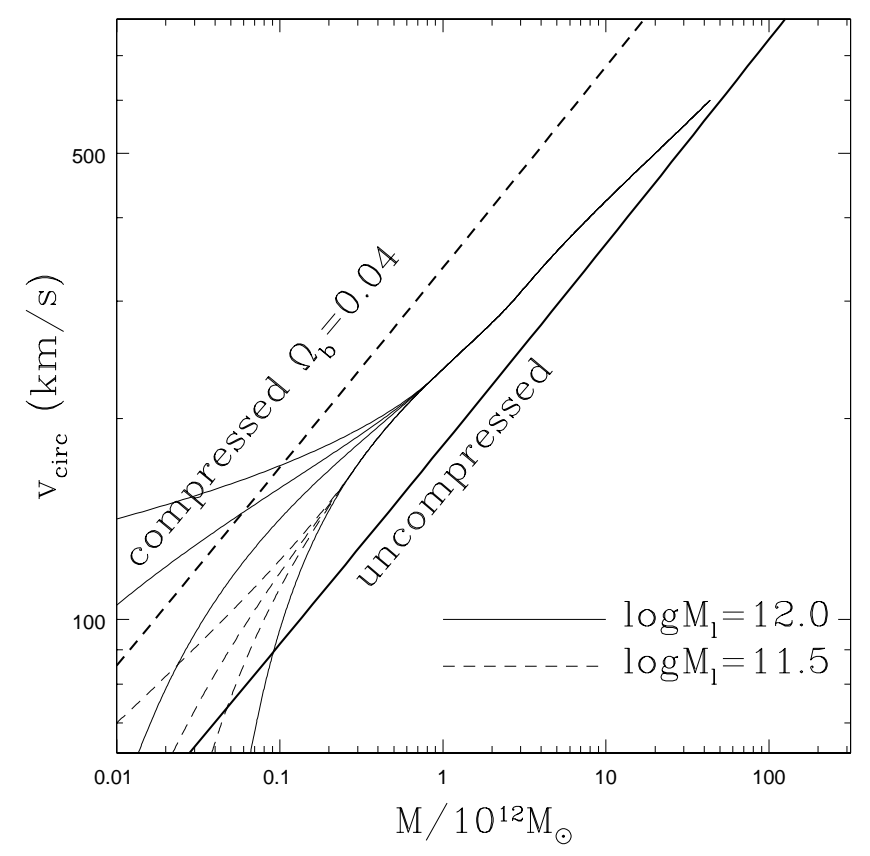

FIGURE 13. The effect of the low mass cutoff in the galaxy formation probability on $v_{c}(M)$. The light solid and dashed lines show the $v_{c}(M)$ relations needed to produce the observed velocity function given low-mass cutoffs at $\log M_{l} / M_{\odot}=11.5$ (dashed) and 12.0 (solid) for power law exponents of $\delta_{l}=1$ (top), $3 / 4,1 / 2$, and 0 (bottom). The case $\delta_{l}=0$ corresponds to having no low-mass cutoff and the two $M_{l}$ cases overlap. The upper cutoff is fixed to $M_{h}=10^{12.5} M_{\odot}$ with $\delta_{h}=1.0$. The heavy solid and dashed lines are the lower and upper bounds corresponding to the uncompressed and the maximally compressed limits of the model.

$M_{h}$. For any set of parameters for $p_{g}(M)$ we can derive the $v_{c}(M)$ required to produce the velocity function.

Figs. 12-14 illustrate the effect of adjusting the structure of the formation probability on the mass-velocity relation $v_{c}(M)$. We start with the high-mass cutoff $\left(M_{h}\right.$ and $\left.\delta_{h}\right)$ since we are certain it exists in order to create the distinction between galaxies and groups/clusters. Physical models, where $v_{c}(M)$ stays within its physical bounds, require a slightly blurred boundary with $\delta_{h} \simeq 1$ and $M_{h} \simeq 10^{12.5} M_{\odot}$ (which we adopt as our standard). Steeper slopes $\delta_{h}$ allow modestly higher mass scales $M_{h}$ but the mass where $p_{g}(M)=1 / 2$ stays roughly in the range $10^{12.5} M_{\odot}$ to $10^{13} M_{\odot}$. The group velocity function is set by $1-p_{g}(M)$ with a velocity set by that of uncompressed halos, $v_{c}(M)=v_{\text {mod, } 0}(M)$. It is the lack of this multi-valued region in our models from $\S 2$ which leads to the "kink" in our model of the velocity function near $v_{c} \sim 400 \mathrm{~km} \mathrm{~s}^{-1}$ (see Fig. 9).

Without a low-mass cutoff in the formation probability, $v_{c}(M)$ would have to rise exponentially with mass in order to convert the steep slope of the mass function into the shallower slope of the velocity function (see Fig. 9). Since this rapidly requires velocities well below that of the halos, we are forced to introduce a low-mass cutoff $\left(M_{l}\right.$ and $\left.\delta_{l}\right)$ to the formation probability. The effect of changing various parameters is shown in Fig. 13 , and we adopt $M_{l}=10^{11.5} M_{\odot}$ and $\delta_{l}=3 / 4$ as our standard model. All models which allow $v_{c}(M)>v_{\text {mod }, 0}(M)$ down to $30 \mathrm{~km} \mathrm{~s}^{-1}$ have a formation probability near $50 \%$ at $10^{11} M_{\odot}$ and $10 \%$ near $10^{10} M_{\odot}$. We introduced these models for $p_{g}(M)$ to see if we could produce a broad mass range in which the formation probability was unity. Fig. 11 


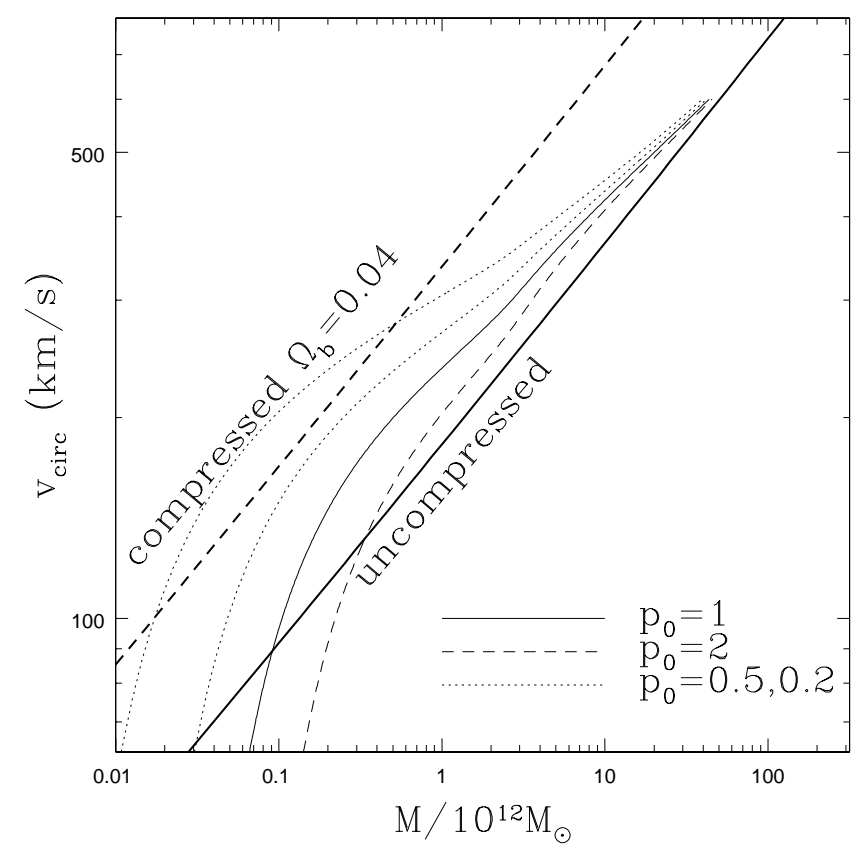

FigURE 14. The effect incompleteness or normalization errors on $v_{c}(M)$. The light curves $v_{c}(M)$ for $p_{0}=2$ (dashed), $p_{0}=1$ (solid), $p_{0}=1 / 2$ (dotted) and $1 / 5$ (dotted) using the standard high mass cutoff. The heavy solid and dashed lines are the lower and upper bounds corresponding to the uncompressed and the maximally compressed limits of the model.

superposes the standard model we derived on the models for $p_{g}(M)$ found without any complicated structure to $v_{c}(M)$. The structure of the functions is nearly identical - the best we can manage is to keep the formation probability unity over one decade in halo mass. From this we conclude that the structure of the velocity function is dominated by the probability $p_{g}(M)$ of forming (or finding) a galaxy rather than variations in the relationship between halo mass and galaxy circular velocity $v_{c}(M)$.

Finally, in Fig. 14 we explore the effects of varying $p_{0}$, which models either errors in the normalization of the mass function or the completeness of the survey underlying the velocity function. We use the standard high mass cutoff and no low mass cutoff. For $p_{0}<1$, which corresponds to making the velocity function survey incomplete or lowering the normalization of the mass function, the velocity corresponding to a given halo rises, and for $p_{0}>1$, which corresponds to raising the normalization of the mass function, the velocity falls. From this we can infer that the velocity function galaxy sample cannot be massively incomplete and the halo multiplicity function cannot differ greatly from unity.

All these conclusions are very similar to the results from attempts to estimate the halo occupation numbers needed to match the halo distributions found in simulations to the observed distribution of galaxies (e.g. Peacock \& Smith 2000, Scoccimarro et al. 2001). For example, Fig. 11 also shows the probability of a halo forming one, two or many galaxies in one of the models by Scoccimarro et al. (2001) based on semianalytic models and matching to large scale structure. The two distributions are very similar in structure given that the Scoccimarro et al. (2001) model has a different normalization.ff

$\dagger$ When using the Scoccimarro et al. (2001) models to populate the halos from an N-body 


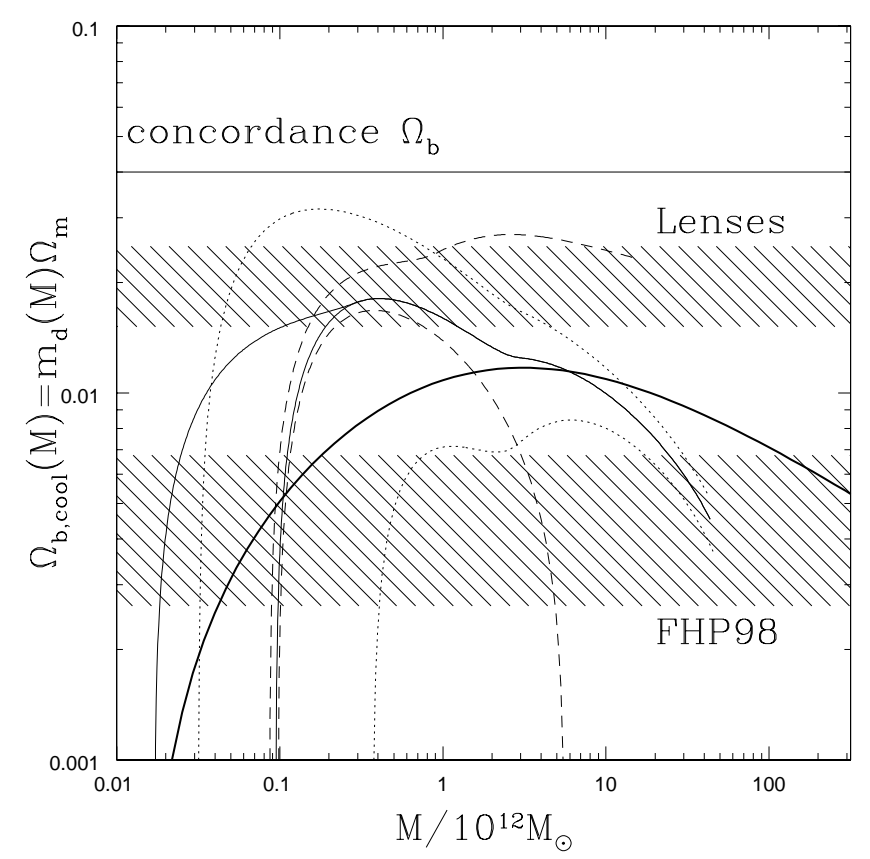

FiguRE 15. Cold baryon densities $\left(\Omega_{b, \text { cool }}=m_{d} \Omega_{m}\right)$. For a range of models of the galaxy formation probability, we use our adiabatic compression models with $\lambda=\bar{\lambda}=0.05$ and $j_{d} / m_{d}=1$ to convert $v_{c}(M)$ into an estimate for the cold baryon density. The light solid curves are for a model with our standard high mass cutoff $\left(\log M_{h} / M_{\odot}=12.5, \delta_{h}=1\right.$ ) with (upper curve at low mass) or without (lower curve at low mass) the standard low mass cutoff $\left(\log M_{l} / M_{\odot}=11.5\right.$, $\delta_{l}=3 / 4$ ) in the formation probability. The dashed lines show consequences of raising the upper cutoff to $\log M_{h} / M_{\odot}=13$ (bottom) or lowering it to $\log M_{h} / M_{\odot}=12$ (top). The dotted lines show the consequences of incompleteness $\left(p_{0}=0.5\right.$, upper $)$ and using too low a mass function normalization ( $p_{0}=2$, lower) including only the standard high mass cutoff and no low mass cutoff. The heavy solid curve shows the cold baryon fraction estimate by Gonzalez et al. (2000) for the Somerville \& Primack (1999) semi-analytic models. The cold baryon density must be less than the total baryon density, $\Omega_{b, \text { cool }}<\Omega_{b}$, and the total baryon density for the concordance cosmological model is marked with the upper horizontal line. The hatched region labeled FHP98 shows the cold baryon density estimated by Fukugita et al. (1998) scaled to the concordance value of $H_{0}$.

Nonetheless, these more sophisticated models for the division of halos into galaxies would greatly improve our more qualitative exploration of the problem.

We finally return to the problem of the dynamical baryon discrepancy. In Fig. 15 we convert some of the allowed mass-velocity relations into estimates of the cold baryon fraction based on the adiabatic compression model scalings (eqns. 2.1 and 2.2). We also show the equivalent relation Gonzalez et al. (2000) derived from the Somerville \& Primack (1999) semi-analytic models. We immediately see that the discrepancy between the estimated baryon content of galaxies (from Fukugita et al. 1998) or the Galaxy (based on the models of Dehnen \& Binney 1998) and the cold baryon fraction needed to compress the halos is present whether we use our simple model of $\S 2$ and the separation distribution of lenses, full semi-analytic models, or totally non-parametric models.

simulation of the cosmological model in $\S 2$ with galaxies, we found that they underestimated the galaxy density by factor of $\simeq 3$. 


\section{The Future}

In this review we examined the relationship between the halo mass function and dynamical probes of it using either the kinematics of galaxies or the separation distribution of gravitational lenses. Although many of the results we discuss are implicit in full semi-analytic models, the approach of examining only the dynamical properties has the advantage of eliminating the dependence of the comparison on luminosity. Dynamical comparisons emphasize the critical role of the cooling baryons in transforming the dynamical structure of halos. The baryonic compression produces a feature in both the distribution of gravitational lens image separations and the local velocity function which is a direct probe of the cold baryon fraction compressing galactic halos. In all our comparisons we find a factor of 2-3 discrepancy between the mass fraction in cold baryons required to explain the data and typical estimates for the cold baryons in galaxies. In a universe with $\Omega_{b} \simeq 0.04$ we need $0.01 \lesssim \Omega_{b \text {,cool }} \lesssim 0.02$ to compress the halos while typical accountings in galaxies find only $0.005 \lesssim \Omega_{b \text {,cool }} \lesssim 0.01$ in known baryonic components. The difference could be explained by MACHOS (see Alcock, Richer and Sahu in these proceedings), cold molecular gas or even warm gas in some circumstances. It is a part of our general problem that we lose track of most of the baryons at redshift zero (see Tripp in these proceedings).

There are three possible solutions to the dynamical baryon discrepancy. First, it could be imaginary - if you select your preferred ranges appropriately you can essentially eliminate the discrepancy. Second, it could be a problem in our models. The adiabatic compression models are crude approximations for the transformation of the dark matter halos by the baryons, and our models have not properly accounted for the halo multiplicity function. There is also a major debate at present about the consistency of observed rotation curves with the predictions of these standard models (see Burkert, Sancisi and Sanders in these proceedings). It is difficult to adequately address this possibility, since it is currently impossible to compute the final structure of a galaxy starting from the initial halo properties without approximations. Third, the accounting for the baryons in galactic halos may be incorrect. The Fukugita et al. (1998) accounting for the baryons in galaxies included only cold gas and normal stellar/stellar remnant populations, neglecting hot gas $\left(10^{6} \mathrm{~K}\right)$, warm ionized gas $\left(10^{4}-10^{5} \mathrm{~K}\right)$, and sub-luminous objects (e.g. MACHOS). While hot gas cannot contribute to the adiabatic compression of the halo, the warm components are both difficult to detect and contribute to the compression.

The most ambitious proposal for the future of the galaxy velocity function is to use its evolution to determine the cosmological equation of state (Newman \& Davis 2000). The challenge here is to understand, control, and then eliminate all the sources of systematic error which can mimic or bias the evolutionary effect. Unfortunately, the evolution is subtle and achieving this goal will be difficult. To put these difficulties in some context, many of the sources of systematic uncertainties are identical to those in the (currently unpopular) attempts to determine the cosmological model using gravitational lens statistics. We can see some of the difficulties in our local comparisons between the halo mass function and the velocity function of galaxies. Different routes to deriving the local velocity function (the choice of surveys, luminosity functions, kinematic relations, type distributions ...), different models for the baryonic mass distribution in galaxies (the distribution of bulge-to-disk ratios, cold baryon fractions, spin parameters.. ) and different models for the connection between halos and galaxies (formation probability, halo multiplicity function ...) all lead to differences that are comparable to the effects of evolution. Our non-parametric analysis shows that at least two one-dimensional functions of the halo mass are needed, the probability $p_{g}(M)$ that a halo of mass $M$ forms a galaxy included 
in the survey, and the average velocity $v_{c}(M)$ of the resulting galaxy, and both of these functions will themselves be evolving and survey-dependent. However, studying the dynamical evolution of galaxies will yield so much information on the evolution of galaxies, that the experiment will be of enormous value even if systematic problems ultimately prevent it from being used for determining the properties of the background cosmology.

Acknowledgments: Much of this work was done in collaboration with M. White ( $\S 2$ and $\S 3)$ or M. Pahre and E. Falco ( $\$ 4$ and $\S 5)$ whose contributions and comments were invaluable. CSK is supported by the Smithsonian Institution and NASA grants NAG58831 and NAG5-9265.

\section{REFERENCES}

Baugh C.M., Cole S., Frenk C.S., 1996, MNRAS, 283, 1361

Benson A.J., et al., 2000, MNRAS, 311, 793

Blanchard A., Sadat R., Bartlett J.G., Le Dour M., 2000, A\&A, 362, 809

Blumenthal G.R., Faber S.M., Flores R., Primack J.R., 1986, ApJ, 301, 27

Browne I.W.A., Myers S.T., 2000, IAU Symposium 201, 47

Bullock J.S., et al., 2000, preprint astro-ph/9908159

Bullock J.S., Dekel, A., Kolatt, T.S., Primack, J.R., \& Somerville, R.S., 2001, ApJ, 550, 21

Cohn J.D., Kochanek C.S., McLeod B.A., Keeton C.R., 2001, ApJ, in press astro-ph/0008390

Cole S., et al., 1994, MNRAS, 271, 781

Cole S., Kaiser N., 1989, MNRAS, 237, 1127

Cole S., Lacey C.G., Baugh C.M., Frenk C.S., 2000, MNRAS, in press astro-ph/0007281

Dalcanton J.J., Spergel D.N., Summers F.J., 1997, ApJ, 482, 659

de Blok, W.J.G., McGaugh, S.S., Bosma, A., \& Rubin, V.C., 2001, astro-ph/0103102

Dehnen, W., \& Binney, J., 1998, MNRAS, 294, 429

Efstathiou, G., Ellis, G., \& Peterson, B.A., 1988, MNRAS, 232, 431

Faber, S.M., \& Jackson, R.E., 1976, 204, 668

Falco, E. E., Kochanek, C. S., \& Munoz, J.A., 1998, ApJ, 494, 47

Flores, R., \& Primack, J.R., 1994, ApJ, 427, L1

Fukugita M., Hogan C.J., Peebles P.J.E., 1998, ApJ, 503, 518

Fukugita M., Turner E.L., 1991, MNRAS, 253, 99

Gonzalez A.H., et al., 2000, ApJ, 528, 145 astro-ph/9908075

Helbig, P., Marlow, D., Quast, R., Wilkinson, P. N., Browne, I. W. A., \& Koopmans, L. V. E. 1999, A\&AS, 136, 297

Hernquist L., 1990, ApJ, 356, 359

Jenkins A., Frenk C.S., White S.D.M. Colberg J.M., Cole S., Evrard A.E., Yoshida N., 2000, MNRAS, in press astro-ph/0005260

Kauffmann G., Colberg J.M., Diaferio A., White S.D.M., 1999, MNRAS, 303, 188

Kauffmann G., White S.D.M., Guiderdoni B., 1993, MNRAS, 264, 201

Keeton, C. R., Falco, E. E., Impey, C. D., Kochanek, C. S., Lehar. J.. McLeod, B. A., Rix, H.-W., Munoz, J. A., \& Peng, C. Y. 2000, ApJ, in press astro-ph/0001500

Keeton C.R., Madau P., 2000, ApJ submitted [astro-ph/0101058]

Keeton C.R., Kochanek C.S., Falco E.E., 1998, ApJ, 509, 561

Keeton C., 1998, Harvard University PhD thesis.

Kitayama T., Suto Y., 1996, ApJ, 469, 480

Kochanek, C. S. 1993a, ApJ, 419, 12

Kochanek C.S., 1995, ApJ, 453, 545

Kochanek C.S., 1996, ApJ, 466, 638

Kochanek C.S., Pahre M.A., Falco E.E., Huchra J.P., Mader J., Jarrett T.H., Chester T., Cutri R., Schneider S.E., 2001, ApJ in press astro-ph/0011456

Kochanek, C.S., \& White, M., 2001, ApJ in press, astro-ph/0102334.

Kochanek, C.S., Pahre, M.A., \& Falco, E.E., 2001, submitted to ApJ, astro-ph/0011458 
Lacey C., Cole S., 1994, MNRAS, 271, 676

Lacey C., Silk J., 1991, ApJ, 381, 14

Li L-X., Ostriker J.P., 2000, preprint astro-ph/0010432

Mahdavi, A., Bohringer, H., Geller, M.J., \& Ramella, M., 2000, ApJ, 534, 114

Maoz, D., \& Rix, H.-W. 1993, ApJ, 416, 425

Maoz D., Rix H.-W., Gal-Yam A., Gould A., 1997, ApJ, 486, 75

Mo H.J., Mao S., White S.D.M., 1998, MNRAS, 295, 319

Moore, B., 1994, Nature, 370, 629

Moore B., et al., 1998, ApJ, 499, L5

Mortlock D.J., Webster R.L., 2000, MNRAS, 319, 872 astro-ph/0008081

Narayan R., White S.D.M., 1988, MNRAS, 231, 97P

Navarro J., Frenk C.S., White S.D.M., 1996, ApJ, 462, 563

Newman J.A., Davis M., 2000, ApJ, 543, L11

Pahre, M.A, Kochanek, C.S., \& Falco, E.E., 2001, in preparation

Peacock, J.A. \& Smith, R.E., 2000, MNRAS, 318, 1144

Pearce F.R., Jenkins A., Frenk C.S., Colberg J.M., White S.D.M., Thomas P.A., Couchman H.M.P., Peacock J.A., Efstathiou G., 1999, ApJ, 521, L99

Phillips P.M.. Browne I.W.A., Wilkinson P.N., Jackson N.J., 2000, IAU Symposium 201 astro$\mathrm{ph} / 0011032$

Porciani C., Madau P., 2000, ApJ, 532, 679

Press W., Schechter P., 1974, ApJ, 187, 425

Rix H.-W., Maoz D., Turner E.L., Fukugita M., 1994, ApJ, 435, 49

Salucci, P., 2001, MNRAS, 320, L1

Scoccimarro, R., Sheth, R.K., Hui, L., \& Jain, B., 2001, ApJ, 546, 20

Sheth R., Tormen G., 1999, MNRAS, 308, 119 (1999)

Shimasaku K., 1993, ApJ, 413, 59

Sigad Y., et al., preprint astro-ph/0005323

Skrutskie, M. F., et al. 1997, in The Impact of Large Scale Near-IR Sky Surveys, F. Garzon et al., eds., (Dordrecht: Kluwer) 187

Somerville R., Primack J., 1999, MNRAS, 310, 1087

Tully, R. B., \& Fisher, B. 1977, A\&A, 54, 661

Turner E.L., Ostriker J.P., Gott J.R., 1984, ApJ, 284, 1

van den Bosch, F.C., Robertson, B.E., Dalcanton, J.J., \& de Blok, W.J.G., 2000, AJ, 199, 1579

van den Bosch, F.C. \& Swaters, R.A., 2001, MNRAS, 325, 1017

Wambsganss J., Cen R., Ostriker J.P., Turner E.L., 1995, Science, 268, 274

Wambsganss J., Cen R., Ostriker J.P., 1998, ApJ, 494, 29

White M., Hernquist L., Springel V., 2001, ApJL, 550, L129

White S.D.M., Frenk C.S., 1991, ApJ, 379, 52

Winn, J. N., Hewitt, J. N., \& Schechter, P. L. 2001, in Gravitational Lensing: Recent Progress, Future Goals, ASP Conf. Series, eds. T. Brainerd \& C. S. Kochanek (San Francisco: ASP) astro-ph/9909335)

Wu X.-P., Xue Y.-J., Fang L.-Z., 1999, ApJ, 524, 22

Wyithe, J.S.B., Turner, E.L., Spergel D.N., 2000, ApJ, submitted astro-ph/0007354 